\title{
Potentials and pitfalls of permafrost active layer monitoring using the HVSR method: a case study in Svallbard
}

\author{
Andreas Köhler ${ }^{1}$ and Christian Weidle ${ }^{2}$ \\ ${ }^{1}$ Department of Geosciences, University of Oslo, Post Box 1047, 0316 Oslo, Norway \\ ${ }^{2}$ Institute of Geosciences, Christian-Albrechts-Universität zu Kiel, Kiel, Germany
}

Correspondence: Andreas Köhler (andreas.kohler@geo.uio.no, andreas.koehler.geo@gmail.com)

Received: 22 June 2018 - Discussion started: 10 July 2018

Revised: 27 November 2018 - Accepted: 15 December 2018 - Published: 10 January 2019

\begin{abstract}
Time-lapse monitoring of the subsurface using ambient seismic noise is a popular method in environmental seismology. We assess the reliability of the horizontal-to-vertical spectral ratio (HVSR) method for monitoring seasonal permafrost active layer variability in northwest Svalbard. We observe complex HVSR variability between 1 and $50 \mathrm{~Hz}$ in the record of a temporary seismic deployment covering frozen and thawed soil conditions between April and August 2016. While strong variations are due to changing noise conditions, mainly affected by wind speed and degrading coupling of instruments during melt season, a seasonal trend is observed at some stations that has most likely a subsurface structural cause. A HVSR peak emerges close to the Nyquist frequency $(50 \mathrm{~Hz})$ in beginning of June which is then gradually gliding down, reaching frequencies of about $15-25 \mathrm{~Hz}$ in the end of August. This observation is consistent with HVSR forward modeling for a set of structural models that simulate different stages of active layer thawing. Our results reveal a number of potential pitfalls when interpreting HVSRs and suggest a careful analysis of temporal variations since HVSR seasonality is not necessarily related to changes in the subsurface. In addition, we investigate if effects of changing noise sources on HVSRs can be avoided by utilizing a directional, narrowband $(4.5 \mathrm{~Hz})$ repeating seismic tremor which is observed at the permanent seismic broadband station in the study area. A significant change of the radial component HVSR shape during summer months is observed for all tremors. We show that a thawed active layer with very low seismic velocities would affect Rayleigh wave ellipticities in the tremor frequency band. We compile a list of recommendations for future experiments, including comments on network layouts suitable for array beamforming and waveform correlation methods that can provide essential information on noise source variability.
\end{abstract}

\section{Introduction}

Environmental seismology is becoming an increasingly popular tool to study earth surface processes and to monitor medium changes in the shallow subsurface through ambient seismic noise analysis (Larose et al., 2015). The latter approach is often based on noise cross-correlation between two receivers which allows the estimation of the medium's Green function under the condition of a random seismic noise source distribution in time and space (Shapiro and Campillo, 2004; Sabra et al., 2005). Continuous seismic noise records therefore do not only allow the inversion of subsurface structures, but also to measure temporal changes therein using seismic noise interferometry (Sens-Schönfelder and Wegler, 2006, 2011; James et al., 2017). An alternative and well-established single-station approach that makes use of ambient seismic noise is the horizontal-to-vertical spectral ratio (H / V spectral ratio or HVSR) technique (e.g., Nakamura, 1989; Lunedei and Malischewsky, 2015; SánchezSesma, 2017, and references therein). Peaks in the HVSR curve are related to strong subsurface seismic velocity contrasts, with shallower interfaces producing higher peak fre- 
quencies. The spectral ratio can be inverted for the shallow subsurface structure based on the diffuse wave field assumption (García-Jerez et al., 2016; Sánchez-Sesma, 2017) or by interpreting it as representing the frequency-dependent Rayleigh wave ellipticity (e.g., Parolai et al., 2005). HVSRs have been shown to be applicable in a wide range of settings, mostly for measuring site resonance frequencies (e.g., Lachet and Bard, 1994) and mapping sediment thickness, but also more recently in the cryosphere to measure ice properties (Lévêque et al., 2010), glacier and ice sheet thickness (Picotti et al., 2017; Yan et al., 2018), or submarine permafrost depths (Overduin et al., 2015). Similar to noise interferometry, the HVSR method does in theory allow time-lapse monitoring of the medium below the station, given that the structural change is significant, a source effect can be ruled out, and the Rayleigh wave ellipticity (or diffuse wave field model parameters) can be extracted precisely enough from the spectral ratios.

It is well known that a seasonally frozen shallow surface layer can affect the site response measured through HVSRs (Xu et al., 2010; Cox et al., 2012). Guéguen et al. (2017), for example, reported a several-day-long HVSR amplitude decrease between 2 and $10 \mathrm{~Hz}$ during an air temperature drop below $0{ }^{\circ} \mathrm{C}$ in Grenoble, France. Furthermore, more recently, a few studies interpreted seasonal changes and emerging peaks in HVSRs at higher frequencies as being the result of the thawing-freezing cycle of the permafrost active layer (Abbott et al., 2016; Kula et al., 2018). HVSRs therefore could bear the potential to become a low-cost, passive, and non-invasive method for long-term monitoring of permafrost with high temporal resolution. However, due to the lack of calibration experiments in the field, to date no standard procedure has been established for such an approach. More studies are needed to explore its limitations and general applicability. For example, a potential pitfall is interpreting HVSR variability as structural change when it is actually due to changes in external site conditions such as noise source distribution and/or meteorological parameters (Chatelain et al., 2008). Violation of the assumption of stationary noise sources might be avoided by using repeating and localized seismic sources, similar to repeating earthquakes that are being used for coda wave interferometry (Snieder, 2006). Environmental seismological research has identified a vast amount of such sources (Larose et al., 2015), e.g., river noise (Burtin et al., 2011), tremors in the cryosphere (Bartholomaus et al., 2015), and anthropogenic structures (Saccorotti et al., 2011; Neuffer and Kremers, 2017).

In this study, we explore the potential of the HVSR method for permafrost active layer monitoring using continuous seismic noise records of several months from a temporary seismic deployment close to Ny-Ålesund on the Arctic archipelago of Svalbard (Fig. 1). We analyze and compare observed seasonal HVSR variability with forward-modeled changes expected from a thawed soil layer using the diffuse wave field theory. Furthermore, we analyze HVSR changes
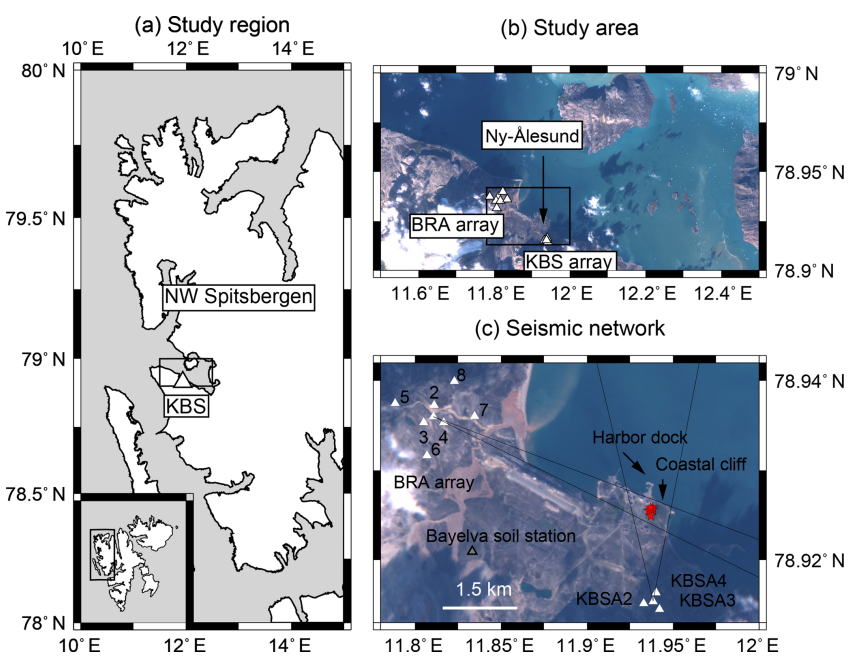

Figure 1. Study area, location of instrumentation, and seismic tremor source. (a) Map of northwest Spitsbergen, part of the Arctic archipelago of Svalbard (lower left corner), and location of permanent seismic station KBS. (b) Study area around Ny-Ålesund and location of temporary BRA and KBSA arrays. The black rectangle shows map section in panel (c). (c) More detailed location of seismic stations and a coastal cliff with shallow cave shown in Fig. 5d being the source of a repeating seismic tremor (see Sect. 5). Red stars are tremor locations between April and August 2016. Black lines indicate azimuthal measurement uncertainty when using FK analysis independently on both arrays. Center station of BRA array is BRA1. Numbers indicate the other instrument locations. Background images: Copernicus Sentinel data 2016.

of a periodically occurring, localized seismic signal which has been present in the record of the permanent seismometer in Ny-Ålesund in all available records since 2001. Finally, we discuss the results and compile a list of recommendations for future field experiments from the lessons learned in our study.

\section{Data}

The permanent Global Seismic Network (GSN) and GeoForschungsNetz (GEOFON) station Kings Bay (KBS) (network codes IU/GE) is located $1.2 \mathrm{~km}$ outside of the settlement of Ny-Ålesund (Fig. 1a-b) within a subsurface $2 \mathrm{~m} \times 2 \mathrm{~m}$ wide and about $2.5 \mathrm{~m}$ deep concrete shelter. Only the channels recording with $40 \mathrm{~Hz}$ sampling (broadband, high gain $(\mathrm{BH})$ channels) are used. The $100 \mathrm{~Hz}$ data (high broadband, high gain $(\mathrm{HH})$ channels) are available in trigger mode only; i.e., solely transient seismic signals unsuitable for noise analysis are being recorded. Between 12 April and 4 September 2016, a temporary seismic network was deployed in the vicinity of Ny-Ålesund (Fig. 1b-c). The deployment consisted of two small-aperture seismic arrays built from $114.5 \mathrm{~Hz}$ three-component geophones connected to Omnirecs DATA-CUBE data loggers, operating with a 
sampling frequency of $100 \mathrm{~Hz}$. The Brandal (BRA) array array (eight stations) was deployed about $2.8 \mathrm{~km}$ northwest of the settlement with an inter-station spacing of about $140 \mathrm{~m}$ (inner ring) and $500 \mathrm{~m}$ (outer ring), and three stations were distributed at about $120 \mathrm{~m}$ distance around KBS (KBSA array). During installation, small holes were drilled into the frozen ground to accommodate the geophone pins. Instruments were covered first with sand and then buried under a rock pile (Fig. A1). Ground coupling of the instruments degraded during melt season and tilting occurred which increased noise levels in almost all records. The stations were revisited on 25 August. While the three temporary stations of the KBSA array were removed, the coupling and leveling of the BRA array instruments were restored, and data were recorded for 10 more days. Note that the temporary deployment was originally not designed as an active layer monitoring experiment but for monitoring iceberg calving at nearby glaciers (Köhler et al., 2016). Similar to most seismic stations (Bonnefoy-Claudet et al., 2006), the seismic noise wave field measured on our network is mainly composed of ocean microseisms at low frequencies $(<1 \mathrm{~Hz})$ and a mixture of (here limited) cultural noise from the close settlement of Ny-Ålesund and effects of local meteorological conditions (wind, ocean swell at local coastline) at high frequencies $(>1 \mathrm{~Hz})$. Frequent calving activity at nearby tidewater glaciers during summer and autumn (Köhler et al., 2015, 2016) mainly affects intermediate frequencies between 1 and $10 \mathrm{~Hz}$.

\section{HVSRs from ambient seismic noise}

We compute daily averaged amplitude spectra for the vertical and horizontal components for all stations. Each continuous daily seismic record is divided into $15 \mathrm{~min}$ long time windows, and the median of the absolute values of the corresponding Fourier spectra is computed. Spectra are smoothed by convolution with a boxcar function (width: 1000 frequency samples with $\mathrm{d} f=0.0038 \mathrm{~Hz}$ ). The horizontal spectra are computed from the north and east components as $\sqrt{\text { north } \times \text { north }+ \text { east } \times \text { east }}$ before computing the spectral ratios. Figures 2 and 3 show results for a selection of stations together with daily air temperature, soil temperature at $0.39 \mathrm{~m}$ depth at a nearby borehole (Boike et al., 2018), and wind speed measured in Ny-Ålesund (see Figs. A2 and A3 for the rest of the stations).

Spectra and HVSRs between April and the beginning of September show complex variability. Spectral amplitudes and HVSRs increase strongly in the course of a few days between the middle and end of May when air temperatures begin to stay above $0^{\circ} \mathrm{C}$. This does not happen simultaneously at all stations (e.g., earlier for KBSA2 and BRA2). Furthermore, high wind speed correlates well with high spectral amplitudes during melt season and with short-term HVSR changes (mostly higher-amplitude ratios). Stations KBSA2,

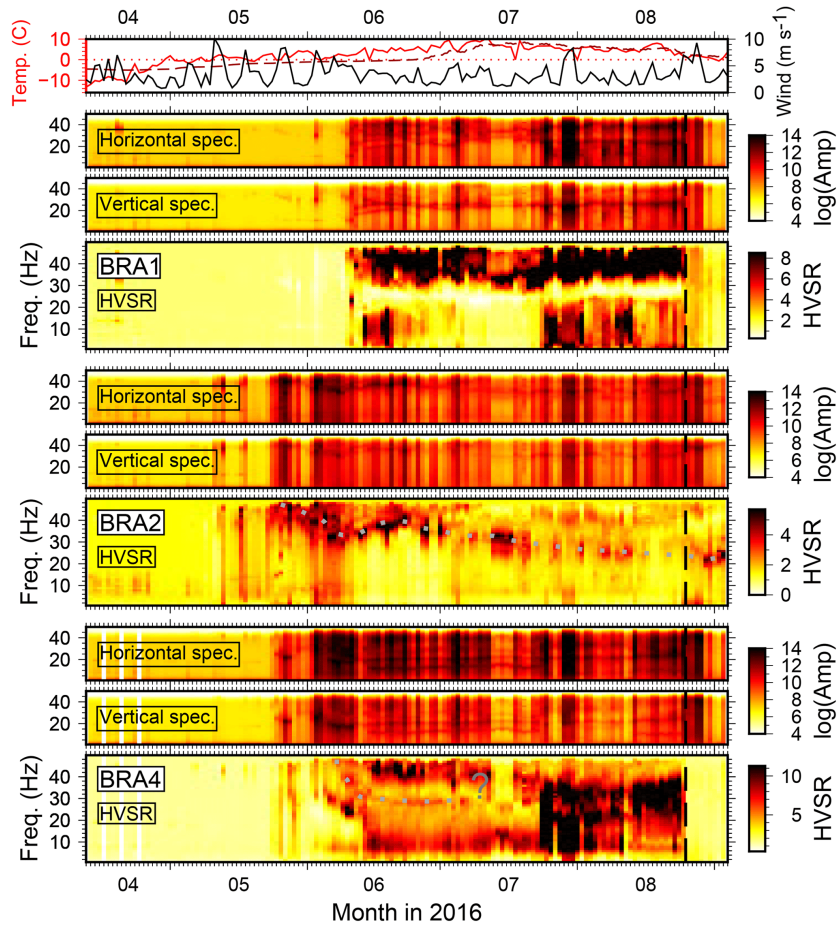

Figure 2. Vertical and horizontal component spectral amplitudes and HVSRs at three stations of the temporary deployment. Dotted lines indicate trend of gliding peak frequencies, question marks ambiguous or unclear peaks, and vertical dashed line date of instrument maintenance (BRA array) or removal (KBSA array). Air temperature (red) and daily averaged wind speed (black) measured in Ny-Ålesund are shown on top. The dashed dark red line is soil temperature at $0.39 \mathrm{~m}$ depth at the Bayelva permafrost observation site (Boike et al., 2018) at $1.6 \mathrm{~km}$ distance from BRA and $2.4 \mathrm{~km}$ from KBS.

KBSA4, BRA2, BRA4, and BRA5 show long-term HVSR trends, i.e., a weak, sometimes diffuse, spectral peak apparently gliding from high frequencies $(50 \mathrm{~Hz})$ in the beginning of June towards low frequencies in the end of August (15$25 \mathrm{~Hz}$ ). However, wind-related short-term HVSR variability is often stronger than, and therefore masking, this long-term trend. At stations KBSA2 and BRA2, the gliding peak trend can be better followed on days of low wind speed. Even if no clear (gliding) peak frequency can be observed over the whole measurement period, stations BRA7 and BRA8 exhibit a strong maximum at $30 \mathrm{~Hz}$ for several days during a calm period in mid-July (Figs. A2 and A3). Most stations of the BRA array show a clear change in the HVSRs after maintenance on 25 August. For example, for BRA2, the gliding frequency peak becomes more pronounced. At BRA1 and BRA4, HVSR amplitudes decrease at all frequencies, while at BRA3 (Fig. A2) a new peak emerges. In addition to the gliding peak at higher frequencies, stations BRA5 and KBSA4 show another weak HVSR peak between 10 and $20 \mathrm{~Hz}$ which also seems to have a slight temporal variabil- 


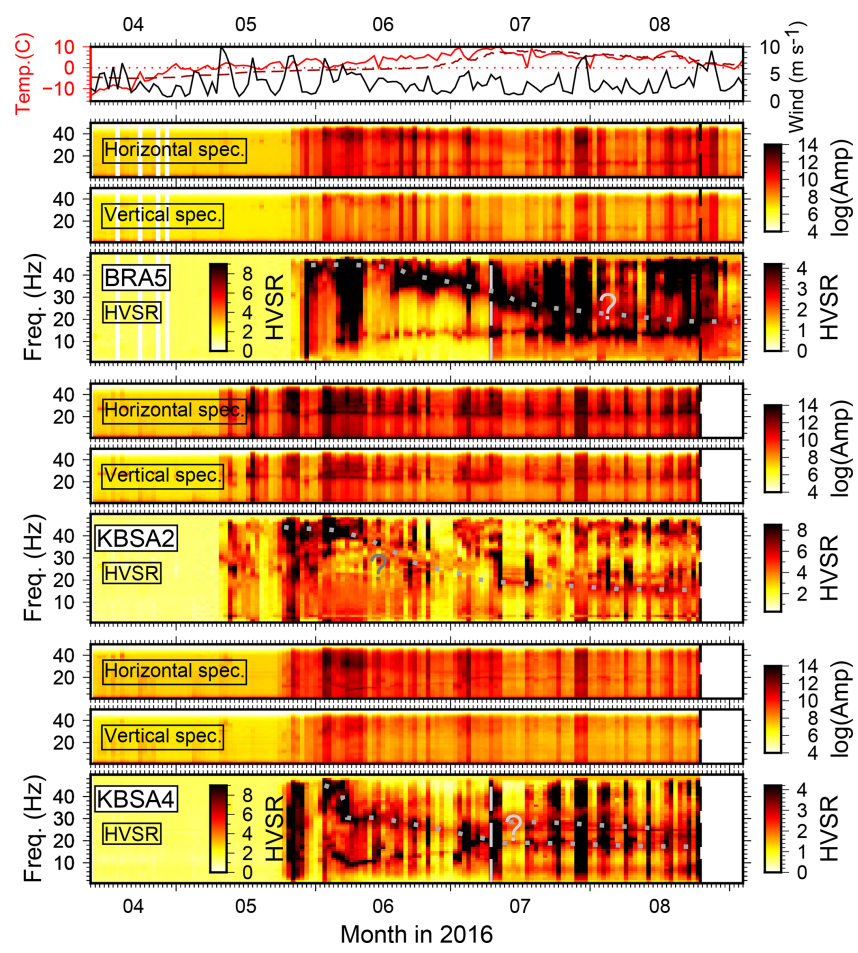

Figure 3. Same as Fig. 2 for three more stations. The gray dashed vertical line indicates change of color scale on 10 July. Color scale is clipped at high HVSRs (black) for BRA5 and KBSA4 to enhance visibility of the weak gliding peak on days of low wind speed. The scale used before 10 July is provided to the left.

ity in June (decreasing and increasing peak frequency). In contrast to the temporary stations, a HVSR peak is observed at KBS close to $20 \mathrm{~Hz}$ with amplitudes correlating well with wind speed but without clear seasonal variations (Fig. A3).

These observations clearly suggest that HVSR variability in our records is complex and cannot merely be explained by a single process such as a structural change in the shallow subsurface. The general increase of seismic noise at the onset of and during the melt season is probably mostly due to flowing water and wind. The variability reflects local noise conditions at each individual station affected by topography, vicinity to streams (BRA1, BRA5, and BRA7), exposure to wind, and extent and timing of degrading instrument coupling related to the progress of snow and soil thawing. Stronger correlation with wind speed is probably due to vibration of the instrument losing coupling, which also affects HVSR amplitudes. Hence, HVSRs do not represent the site response during these time periods. The short-term HVSR variability is therefore not related to a structural change and frequency peaks not necessarily to subsurface interfaces. However, the long-term trend (gliding peak frequency) cannot be easily explained by changing noise conditions and is most likely related to a structural change such as the increasing thaw depth below the station (see discussion below). In fact, the onset of the gliding coincides well with the soil temperature at $0.39 \mathrm{~m}$ depth reaching $0{ }^{\circ} \mathrm{C}$. When instrument vibrations dominate and/or ground coupling is too degraded, this structural effect seems to be too weak to be visible during particular time periods or during the entire record for some stations (e.g., BRA1, BRA4). When coupling is restored, strong, nonstructural HVSR amplitude peaks disappear (BRA1, BRA4) and/or HVSR peaks, presumably due to subsurface structure, are more clearly revealed (BRA2).

\section{Modeled HVSRs}

In order to evaluate the effect of the permafrost active layer, we model HVSRs for a series of subsurface seismic velocity models using the diffuse wave field theory, which takes into account surface and body waves (HVInv, García-Jerez et al., 2016; Sánchez-Sesma, 2017). The thaw depth in the $\mathrm{Ny}$-Ålesund area can reach up to $2 \mathrm{~m}$ in summer (Westermann et al., 2010). The total permafrost depth is between 100 and $150 \mathrm{~m}$ (Haldorsen et al., 1996; van der Ploeg et al., 2012). The seismic $S$-wave velocity change in the active layer is significant, ranging from 0.1 to $0.5 \mathrm{~km} \mathrm{~s}^{-1}$ in unfrozen wet soil, depending on liquid water saturation, to $0.9-2.5 \mathrm{~km} \mathrm{~s}^{-1}$ in frozen conditions (e.g., King et al., 1988; LeBlanc et al., 2004; Cox et al., 2012; James et al., 2017). We use a 1-D subsurface velocity reference model (Table 1 ) inspired by the geological information available (e.g., Fig. 4 in Haldorsen and Heim, 1999). We modify the model by introducing an active layer of different thickness $(0-2.5 \mathrm{~m})$ and seismic $S$-wave velocity $\left(\mathrm{Vs}=0.1-1.0 \mathrm{~km} \mathrm{~s}^{-1}\right)$ to simulate different stages during the thawing process (Fig. 4a-c). The active layer thickness is either fixed and seismic velocity is being decreased stepwise, or the seismic velocity is fixed and the thaw depth is increased successively. The latter model is presumably closer to the real situation; however, there might also be a gradual warming/thawing of the soil from top to bottom leading to a decreasing effective seismic velocity in the active layer over time. In addition, we correct the modeled HVSRs with the instrument response of the geophone to simulate the effect of the anti-aliasing filter at the Nyquist frequency $(50 \mathrm{~Hz})$.

As expected, results show the emergence of a HVSR peak related to the increasing or deepening velocity contrast in the shallow subsurface. The peak frequency decreases to values between 12 and $20 \mathrm{~Hz}$ for maximum thaw depths, depending on how low the $S$-wave velocity is assumed to drop. Spectral ratio amplitudes are affected down to $5 \mathrm{~Hz}$. Due to the upper frequency limit at $50 \mathrm{~Hz}, \mathrm{HVSR}$ peaks begin to emerge below the Nyquist frequency at about $35 \mathrm{~Hz}$, increase in amplitude (Fig. 4c), and then glide towards lower frequencies if the $S$ wave velocity decreases below $0.3 \mathrm{~km} \mathrm{~s}^{-1}$ (Fig. 4b).

The contribution of Love waves in the ambient noise depends on site conditions and affects the amplitude of the HVSR peak but in most cases does not change the peak frequency itself (Bonnefoy-Claudet et al., 2008). Furthermore, noise source characteristics can lead to variations in 
Table 1. Reference seismic velocity models for the study site based on geological site information available (Haldorsen and Heim, 1999) and adjusted to explain observed Rayleigh wave ellipticities and phase velocities. Winter model: frozen active permafrost layer. Summer model: unfrozen active layer. HS: half space. Geological units in Haldorsen and Heim (1999): U1: sandstone, U2: shale, U3: chert, glauconitic sandstone, U4: dolomite, limestone, U5: basement. ACL: thawed active layer. Vp: seismic $P$-wave velocity. Vs: seismic $S$-wave velocity.

\begin{tabular}{|c|c|c|c|c|c|c|c|c|}
\hline \multicolumn{4}{|c|}{ Winter model } & \multicolumn{4}{|c|}{ Summer model } & \multirow[t]{2}{*}{ Unit } \\
\hline $\begin{array}{l}\text { Thickness } \\
\text { (m) }\end{array}$ & $\begin{array}{r}\mathrm{Vp} \\
\left(\mathrm{km} \mathrm{s}^{-1}\right)\end{array}$ & $\begin{array}{r}\mathrm{Vs} \\
\left(\mathrm{km} \mathrm{s}^{-1}\right)\end{array}$ & $\begin{array}{r}\text { Density } \\
\left(\mathrm{g} \mathrm{cm}^{-3}\right)\end{array}$ & $\begin{array}{r}\text { Thickness } \\
(\mathrm{m})\end{array}$ & $\begin{array}{r}\mathrm{Vp} \\
\left(\mathrm{km} \mathrm{s}^{-1}\right)\end{array}$ & $\begin{array}{r}\mathrm{Vs} \\
\left(\mathrm{km} \mathrm{s}^{-1}\right)\end{array}$ & $\begin{array}{r}\text { Density } \\
\left(\mathrm{g} \mathrm{cm}^{-3}\right)\end{array}$ & \\
\hline & & & & 2 & 1.0 & 0.1 & 1.5 & ACL \\
\hline 90 & 2.5 & 1.0 & 2.0 & 88 & 2.5 & 1.0 & 2.2 & $\mathrm{U} 1 / \mathrm{U} 2$ \\
\hline 37 & 3.0 & 1.35 & 2.2 & 37 & 3.0 & 1.35 & 2.2 & $\mathrm{U} 3$ \\
\hline 123 & 5.0 & 3.0 & 2.4 & 123 & 5.0 & 3.0 & 2.4 & $\mathrm{U} 3$ \\
\hline 350 & 6.0 & 3.5 & 2.7 & 350 & 6.0 & 3.5 & 2.7 & U4 \\
\hline HS & 6.4 & 3.8 & 3.0 & HS & 6.4 & 3.8 & 3.0 & U5 \\
\hline
\end{tabular}
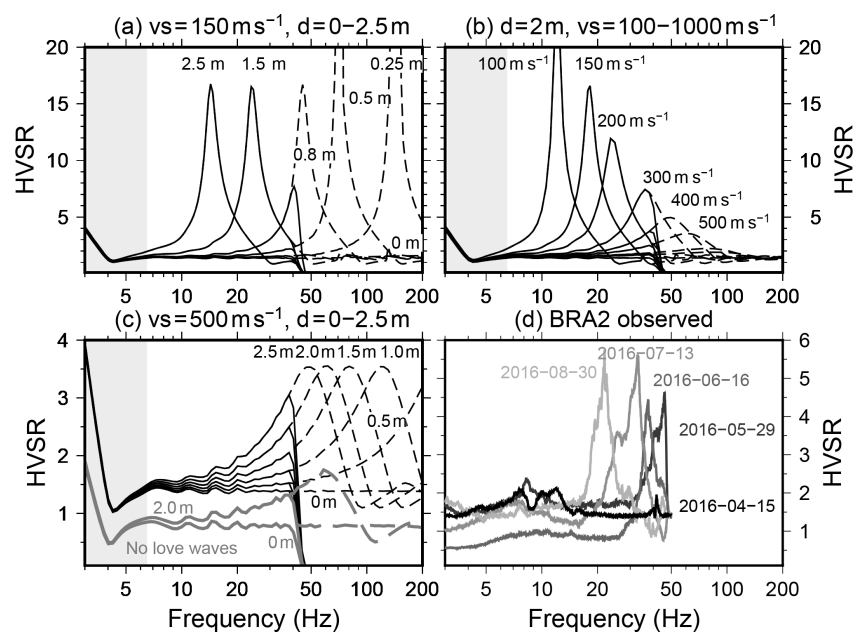

Figure 4. (a-c) HVSRs modeled using the diffuse wave field method and subsurface models of increasing thaw depth $d$ or decreasing $S$-wave velocity Vs in the active layer. The reference model in Table 1 is modified accordingly. Black models include Rayleigh, Love and body waves. Gray models in panel (c) include no Love waves. The gray area indicates tremor frequency band (see Sect. 5). Dashed curves are modeled HVSRs above the Nyquist frequency without using the anti-aliasing filter of field instruments. (d) Measured HVSRs at station BRA2 on four different days showing a peak gliding to lower frequencies.

the fraction of Love waves (Köhler et al., 2006). In case Love waves are excluded from our forward computation, the HVSR amplitudes are significantly lower compared to the full diffuse wave field; however, the peak frequency is unaffected (Fig. 4c). The amplitude differences between models including and excluding Love waves are of the same order as amplitude variations for apparent peaks resulting from velocity reduction or thaw depth increase close to the Nyquist frequency.

\section{HVSRs from a repeating seismic tremor}

For better discriminating the causes of HVSR variability, analysis could be restricted to seismic records of a particular localized, repeating, and directional noise source. Furthermore, observations within longer time periods are essential to validate the HVSR seasonality observed above. However, since the permanent station KBS has a lower sampling rate, we cannot resolve the relevant frequency range above $20 \mathrm{~Hz}$. Furthermore, since the about $2.5 \mathrm{~m}$ deep KBS shelter sits on permanently frozen soil, the effect of active layer variability on HVSRs is expected to become smaller at higher frequencies since decreasing wavelengths sense less of the surrounding medium and more of the concrete shelter. This could explain the lack of a clear HVSR seasonality close to $20 \mathrm{~Hz}$ (Fig. A3). However, this might be different if a dominant contribution of seismic signals with longer wavelengths exists. In fact, we observe such a signal at KBS and explore its potential to resolve active layer changes.

\subsection{The tremor}

A characteristic feature at KBS is a pronounced change in the character of ambient seismic noise during certain time periods all year round and in all available records from 2001 to 2016 (except for data gaps between 2001 and 2004). A tremor-like signal occurs, typically lasting for about several hours (Figs. 5a and B1) in a narrow frequency band between 3 and $6 \mathrm{~Hz}$, with a temporally stable spectral peak on the vertical component at $4.5 \mathrm{~Hz}$ (Fig. 5c). A remarkably clear semi-diurnal occurrence pattern is observed in the temporal distribution of spectral amplitudes which correlates well with the sea level measured in Ny-Ålesund (Fig. 5a). We will refer to this signal as a "repeating tremor" or simply "tremor".

We detect repeating tremors automatically in the entire available KBS record using a short-time over long-time average (STA/LTA) trigger algorithm applied to a time series of vertical component spectral amplitudes (see Appendix B for details). All tremor detections between 2001 and 2016 occur 
(a) Examples of tremor occurrences

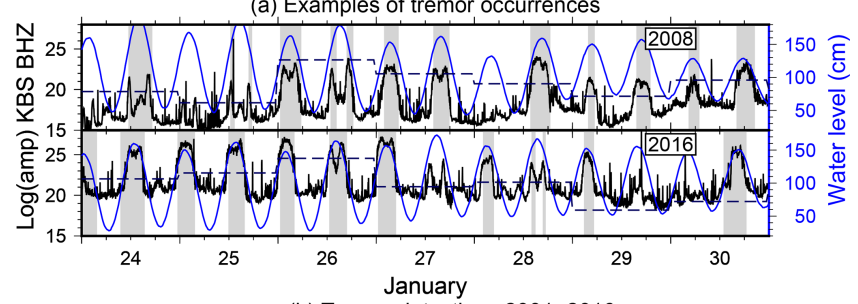

(b) Tremor detections 2001-2016

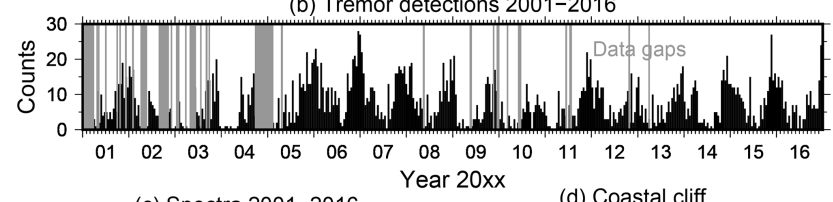

(c) Spectra 2001-2016
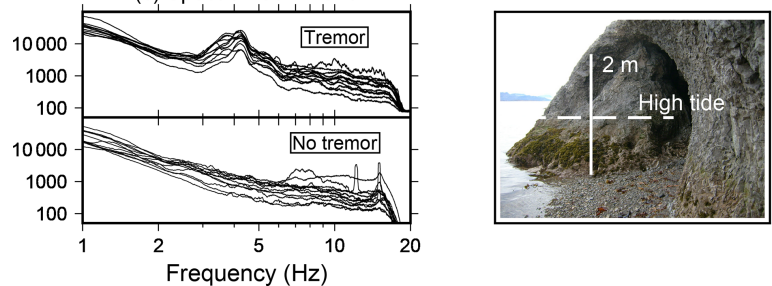

Figure 5. Repeating seismic tremor measured at KBS. (a) Temporal distribution of spectral amplitude between 3.4 and $5.7 \mathrm{~Hz}$ and water level (chart datum) at the end of January in 2008 and 2016. The high spectral power lasting several hours are tremor time periods which correlate with ocean tides. Gray areas indicate automatic tremor detections. Horizontal dashed lines show the relative change in daily wind speed. (b) Temporal distribution of seismic tremor detections (counts in 2 weeks). (c) Monthly averaged amplitude spectra of seismic tremor detections (vertical component) and of a selection of monthly time periods without tremors (2016 only). (d) Suggested tremor source: coastal cliff with shallow marine cave (Fig. 1c).

around semi-diurnal tidal maxima in Ny-Ålesund. However, during neap tides and low wind speeds, almost no tremors are detected (see average daily wind speed in Fig. 5a). The Fourier transform of the time series of log-spectral powers used for the detector fits remarkably well with the ocean tide spectrum and therefore confirms tidal modulation (Fig. B2). Furthermore, the number of tremors varies seasonally, with more detections from late summer to late spring (Fig. 5b).

We use the temporary KBS and BRA arrays to locate tremors which occurred during the deployment period in 2016 by means of frequency-wavenumber analysis (FK; Kvaerna and Ringdal, 1986; Ohrnberger et al., 2004) and the spatial mapping by multi-array beamforming method (SMAB, in the Supplement of Köhler et al., 2016). Figure 1c shows that the tremor source is spatially stationary and very localized at the shoreline in the area of the harbor of $\mathrm{Ny}$ Alesund. Location accuracy is limited because of the resolution limit of array beamforming given the tremor wavelength (about $400 \mathrm{~m}$ ). A possible source location is a $270 \mathrm{~m}$ long and 3-4 m high cliff with a shallow cave-like opening at $200 \mathrm{~m}$ distance to the east of the harbor (Fig. 5d). Another poten- tial source is the harbor dock, a grounded artificial structure with an extent of about $100 \mathrm{~m}$. However, ocean wave activity should cause vibration of the dock at high as well as low tides, unless an unknown mechanism causes vibrations only if the water level reaches the upper part of the structure. We therefore have more evidence for the cliff at the marine cave being the source of the tremor. A reasonable source mechanism for the tremor signal is therefore slamming of breaking sea waves at the cliff during high tides and significant ocean wave activity (Adams et al., 2002; Young et al., 2016), often accompanied by high wind speeds. At low tides and/or high tides during the neap tide cycle, a narrow beach is exposed and the ocean waves do not reach the cliff, which explains the temporal distribution of tremor occurrences. Furthermore, ocean wave activity usually being stronger during autumn and winter and spring tides being strongest around the equinox in March and September, is a good explanation for the seasonality (Fig. 5b). Our observations are consistent with previous studies on ocean wave cliff interaction causing microseismic cliff-top ground motion within a frequency band of 1 to $50 \mathrm{~Hz}$ (Dickson and Pentney, 2012; Norman et al., 2013), with peaks around $10 \mathrm{~Hz}$ (Jones et al., 2015; Earlie et al., 2015) and tidal modulation (Earlie et al., 2015). The slamming forces of breaking ocean waves might be stronger in the cave because of the confined space, which could be an explanation for the signal strength even at $2 \mathrm{~km}$ distance (BRA array). No similar signals are observed from a few other shoreline cliffs in the area which are located between mostly flat beaches.

Beamforming analysis of the vertical components of the KBSA array suggests that the tremor signal consists predominantly of surface waves. Apparent seismic phase velocities show typical dispersion, with values between 1.5 and $2.0 \mathrm{~km} \mathrm{~s}^{-1}$ (Fig. C1b). In contrast to frequencies below $2 \mathrm{~Hz}$ and above $6 \mathrm{~Hz}$, where ambient seismic noise dominates the wave field, the back-azimuth in the tremor frequency range fluctuates only slightly and points clearly to north on average (Fig. C1a).

\subsection{Tremor spectrum and polarization}

We analyze all three spatial wave field components to gain more insight into the propagation properties of the seismic tremor. Figure $6 \mathrm{~b}$ shows the spectral amplitudes of the radial component for a single tremor testing different back-azimuth angles. The spectrum is computed as the median of individual amplitude spectra obtained for 15 min long time windows. The first and last $35 \mathrm{~min}$, where the tremor gradually emerges or disappears, are not analyzed to prevent ambient seismic noise affecting the results. The following results are representative for all other tremors between 2001 and 2016. It is striking that high spectral amplitudes on the horizontal components alternate between the frequency ranges of 3 4 and $4-5 \mathrm{~Hz}$ for different back-azimuth angles, whereas on the vertical component the entire frequency range of $3-5 \mathrm{~Hz}$ 


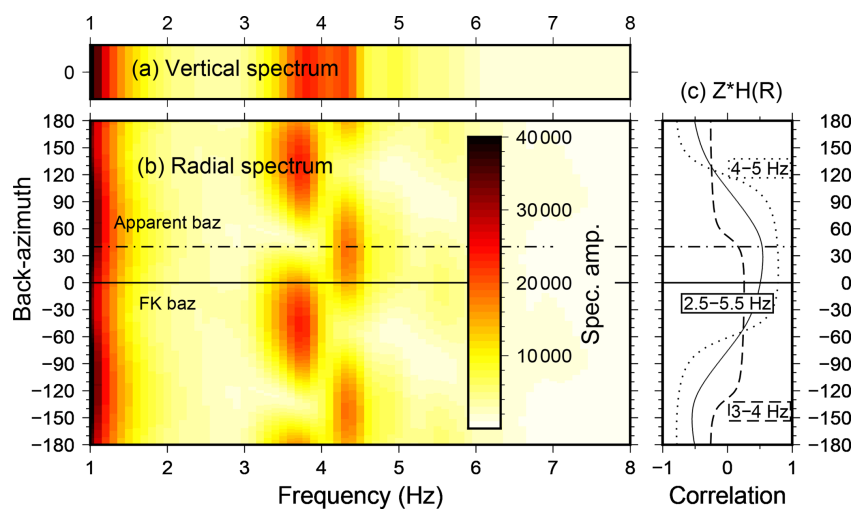

Figure 6. (a, b) Example amplitude frequency spectra at KBS for a tremor occurring between 12 May 2016 03:02:00 UTC and 12 May 2016 06:17:00 UTC for vertical and radial components assuming different back-azimuth (baz) values. (c) Correlation coefficient between vertical and Hilbert-transformed radial component assuming different back-azimuth values in different frequency bands. High values are expected in the case of Rayleigh waves on the radial component. Tremor back-azimuth from vertical FK analysis (FK back-azimuth) and apparent back-azimuth corresponding to maximum correlation between 4 and $5 \mathrm{~Hz}$ (apparent back-azimuth) are indicated. Discrepancy is probably due to azimuthal anisotropy.

dominates (Fig. 6a). Maximum amplitudes in both frequency bands correspond to perpendicular directions which do not coincide with the propagation direction from north to south, as inferred from vertical component FK analysis. In fact, the maximum between 4 and $5 \mathrm{~Hz}$ is about $40^{\circ}$ off the propagation direction.

We evaluate the tremor polarization by cross-correlating the vertical and the Hilbert-transformed radial component. In the case of dominant surface waves, the radial component for a back-azimuth of $0^{\circ}$ (location of tremor source) should yield a pure Rayleigh wave with elliptic polarization. However, according to Fig. 6c, the polarization maximum is clearly shifted towards positive back-azimuth angles between 4 and $5 \mathrm{~Hz}$ coinciding well with the radial component amplitude maximum. On the other hand, correlation of vertical and Hilbert-transformed radial components between 3 and $4 \mathrm{~Hz}$, and thus ellipticity, is very low for all back-azimuth angles. This suggests that Rayleigh waves on the horizontal components only dominate between 4 and $5 \mathrm{~Hz}$ for an (apparent) back-azimuth of about $40^{\circ}$. Furthermore, it seems that Love waves from the same direction dominate between 3 and $4 \mathrm{~Hz}$ since maximum amplitudes are observed for a rotation angle of $130^{\circ}$, the corresponding transverse component. The lack of Rayleigh wave energy on the radial component in this frequency band and the presence on the vertical component can be explained by a trough in the frequency-dependent ellipticity. It remains, however, unclear why Love waves disappear between 4 and $5 \mathrm{~Hz}$.

The back-azimuth discrepancy between vertical FK and polarization analysis may be due to azimuthal anisotropy or a misorientation of the KBS instrument. The latter possibility can be excluded since systematic bias towards positive back-azimuth angles is also observed on the temporary stations of the KBSA array. Furthermore, an analysis of $P$-wave polarization from regional earthquakes at KBS revealed a similar behavior. There is a systematic backazimuth-dependent bias at KBS between polarization angle and expected back-azimuth (Fig. C2). This bias is positive at $0^{\circ}$ back-azimuth. Subsurface geology in the $\mathrm{Ny}$-Ålesund area exhibits southwest-dipping sediment layers (Figs. 3 and 4 in Haldorsen and Heim, 1999) which could give rise to azimuthal anisotropy, i.e., a rotation of the polarization ellipsoid (clockwise from north) with respect to propagation direction (north to south). A quantification and further analysis of this finding are beyond the scope of this paper and should be subject of future studies.

\subsection{Variability of Rayleigh wave ellipticity}

We compute HVSRs of all tremor records at KBS to analyze the Rayleigh wave ellipticity using the same processing as for the ambient noise. Since we found clear evidence that the angle separating Rayleigh and Love waves on the radial and tangential components does not coincide with the propagation direction inferred from the vertical component (Fig. 6) and as suggested by the tremor source location, we compute the radial-to-vertical spectral ratios (RVSRs) using a back-azimuth of $40^{\circ}$. Figure $7 \mathrm{~b}$ shows that the RVSRs are very stable and their standard deviations low within the tremor frequency band. A complex peak-trough shape of the RVSR curve is revealed. After testing different (1-D) subsurface velocity models based on our reference model (Table 1), it turned out that this behavior can only be explained by a mixture of fundamental and higher-mode Rayleigh wave ellipticities (compare Fig. 7a and b). The first trough at $4 \mathrm{~Hz}$ can be related to the ellipticity minimum of the fundamental and first higher modes. The fundamental mode peak below $3 \mathrm{~Hz}$ lays outside the tremor band and is probably therefore not revealed. The first RVSR peak between 4 and $5 \mathrm{~Hz}$ seems to coincide with the first higher-mode ellipticity maximum. The next trough would then be related to an ellipticity minimum which results from the superposition of first and second higher modes. At the upper limit of the tremor band at $6 \mathrm{~Hz}$, another peak could be related to the second highermode peak.

The radial component HVSRs of all tremor occurrences between 2001 and 2016 exhibit very similar shapes (monthly averaged RVSRs are provided in the Supplement S03). However, there is a slight but significant ( $p<0.01$ for equal mean hypothesis in Welch's $T$ test) seasonal variation in the amplitudes between 4.0 and $5.8 \mathrm{~Hz}$ (Fig. 7b). The amplitudes are higher during the summer months between June and September. We quantify the RVSR variability by computing the root mean square (rms) difference between 4.5 and $5.5 \mathrm{~Hz}$ with respect to the average RVSR of tremor records in February 


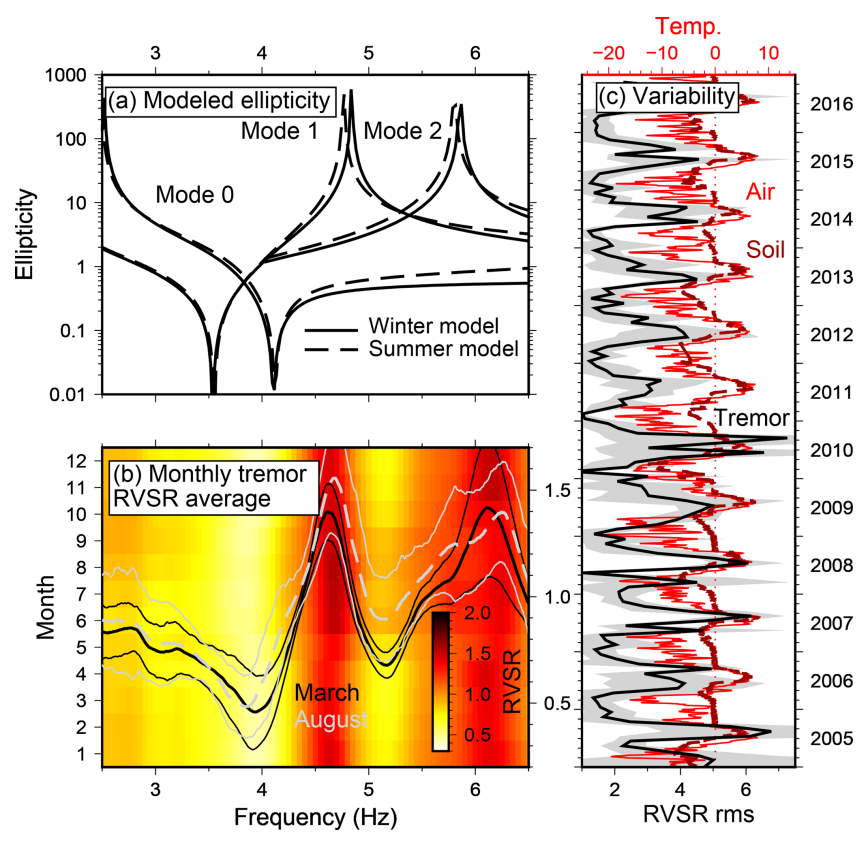

Figure 7. (a) Rayleigh wave ellipticities for fundamental and two higher modes in the tremor frequency band computed from the reference model and modified model by introducing a $2 \mathrm{~m}$ thick top low-velocity layer supposed to represent a thawed active permafrost layer. (b) Temporal variation of tremor radial-to-vertical spectral ratios (RVSRs) averaged over individual months and the years 20102016. Average RVSRs for March and August and standard deviations show that the seasonal change in amplitude is significant and consistent ( $p<0.01$ between 4.0 and $5.8 \mathrm{~Hz}$ for Welch's $T$ test). (c) Air temperature measurements in Ny-Ålesund (10-day running average), soil temperature at $0.59 \mathrm{~m}$ depth (dark red dashed; Boike et al., 2018), and monthly averaged root mean square (rms) difference for frequency range $(4.0-5.5 \mathrm{~Hz})$ between averaged RVSRs in February 2016 and each tremor RVSR. Standard deviations are shown as gray areas. The years 2001-2004 are not shown because of long data gaps.

2016 (Fig. 7c), which reveals a clear seasonality in all years. As soon as air and ground temperatures increase above $0{ }^{\circ} \mathrm{C}$, rms values increase rapidly, before dropping again in autumn when temperatures approach negative degrees.

\section{Discussion of the reliability of HVSRs for permafrost monitoring}

The results of our field measurement and theoretical modeling reveal a number of challenges and pitfalls when attempting to use HVSRs to monitor the active permafrost layer. In the case of ambient seismic noise, the general broadband HVSR amplitude increase and the emergence of amplitude peaks in the beginning and during the melt season could be mistaken for a direct structural effect of the active layer. Furthermore, strong HVSR peaks resulting from short-term changes in the noise sources, e.g., wind affecting the instru- ment directly or generating noise at or at close proximity to the measurement site, could be misinterpreted as HVSR peaks related to subsurface interfaces if the recording period is too short or wind speed measurements are not available. At the same time, a weaker structural peak might be masked by such noise sources. Moreover, an emerging HVSR peak close to the Nyquist frequency could be an artifact of the instrument anti-aliasing filter (Fig. 4c); i.e., it could be related to an emerging peak at higher frequencies and would lead to an overestimation of the thaw depth if the apparent peak is misinterpreted. Furthermore, a frequency-dependent seasonal change of the relative contribution of Rayleigh and Love waves will affect HVSR amplitudes and could give rise to misinterpretation of the caused HVSR variability that is not related to a structural change. Finally, for measuring HVSR changes caused by the active layer, seismic instruments have to be deployed on top of or inside the soil, which naturally leads to degrading coupling, tilt, and/or instrument vibrations during thawing. The processes above include issues known from previous studies to affect HVSRs. For example, Chatelain et al. (2008) mentioned among other effects strong tilt, strong wind when recording next to a feature connected to the ground, and heavy rain. The main focus of Chatelain et al. (2008) was the frequency range below $20 \mathrm{~Hz}$; however, one would expect these issues to become even more relevant at higher frequencies, which is a reason why it was recommended to restrict HVSR analysis to frequencies below $10 \mathrm{~Hz}$. Nevertheless, in order to resolve a HVSR peak caused by the active layer, we need to take these frequencies into account.

Another finding of Chatelain et al. (2008) is strong effects related to the nature of the shallow uppermost layer. Thick $(>10-15 \mathrm{~cm}$ ) mud and ploughed and/or water-saturated soil were shown to lead to higher HVSR amplitudes and appearance of artificial peaks at higher frequencies. Similarly, we have clear indications for a shallow structural variation causing a temporal change in the HVSRs at 5 out of 11 seismic stations and short-term HVSR peaks at two more stations during days of low wind speed that can be attributed to the permafrost active layer (Fig. 8). The gliding frequency peaks are consistent with a realistic active layer thawing process starting in the beginning of June and reaching, consistently with the modeling results, a thaw depth of about $2 \mathrm{~m}$ and $S$-wave velocities between $0.15-0.25 \mathrm{~km} \mathrm{~s}^{-1}$ at the end of the summer. The best example is station BRA2, where a peak emerges in May at $46 \mathrm{~Hz}$ (probably underestimated because of the anti-aliasing filter) from a flat HVSR curve measured in April (Fig. 4d). Subsequently, the peak frequency decreases to $38 \mathrm{~Hz}$ in June, $33 \mathrm{~Hz}$ in July, and $22 \mathrm{~Hz}$ in August. Furthermore, HVSR peak amplitude ratios lay in the range of the modeled values. BRA2 was located at the eastern foot of a small hill, probably shielding the instrument more efficiently from wind coming dominantly from west. Hence, our results suggest that HVSRs can indeed be used to monitor the thawing-freezing cycle in permafrost, given that 


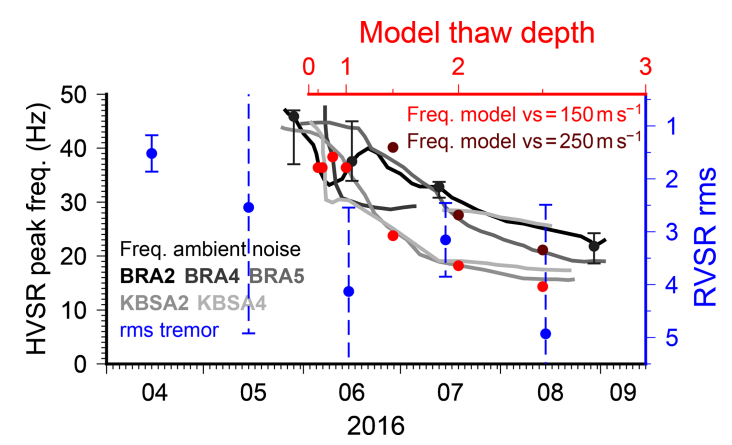

Figure 8. Effect of permafrost active layer on HVSR measurements. Comparison of observed ambient noise HVSR peak frequencies for stations BRA2, BRA4, BRA5, KBSA2, and KBSA4 (solid lines) and modeled peak frequencies (red symbols) taking into account anti-aliasing filter of seismic instrument. For station BRA2, additional black symbols and error bars show peak frequencies and uncertainties corresponding to days in Fig. 4d. For the $x$ axis on top representing modeled thaw depth, we assume a square root dependency with time from the beginning of June. Tremor RVSR rms values from Fig. 7 are shown. The rms and peak frequencies follow a similar trend.

a careful analysis of the temporal variability has been carried out as pointed out above. However, more calibration experiments are necessary to relate peak frequency directly to thaw depth and soil properties, as well as to identify preferable sites for such measurements.

As a special case of the known seasonal effect on HVSRs related to the thawing-freezing cycle (e.g., Guéguen et al., 2017), variability caused by the permafrost active layer has been reported previously (Abbott et al., 2016; Kula et al., 2018). Instead of geophones, Abbott et al. (2016) (same experiment as James et al., 2017) used Posthole sensors buried in the active layer, since these instruments are less sensitive to tilt. Such an instrumentation would therefore eliminate some of the noise issues we face with our deployment. Furthermore, in that study, emerging HVSR peaks between 10 and $30 \mathrm{~Hz}$ were observed during summer, which, however, could not be explained by the relatively shallow active layer thickness of $68 \mathrm{~cm}$ at the study site. Kula et al. (2018) described seasonal HVSR variability at a seismic station in southern Svalbard. Since a permanent station was used with $100 \mathrm{~Hz}$ sampling, higher frequencies were resolved than possible at KBS, and instrument coupling was not an issue. However, similar to our results, the authors acknowledged that low-frequency HVSR peaks (e.g., at $12 \mathrm{~Hz}$ ) and overall seasonal HVSR amplitude increase are due to wind noise and/or human activity at the research station in summer. They also described a peak, but not gliding as in our case, emerging in June at $40 \mathrm{~Hz}$ close to the Nyquist frequency, accompanied by a minimum at $30-35 \mathrm{~Hz}$, which they attribute to active layer thawing. The observations of both previous studies support our conclusion that HVSR interpretation must be done carefully, as strong HVSR peaks or amplitude increases in general are not necessarily related to shallow structural changes, although they appear seasonally.

A station network allows us to pursue different approaches than simply applying the single-station HVSR method. Beside two-station noise interferometry to measure seismic velocity changes (James et al., 2017), array analysis makes it also possible to measure the frequency-dependent ratio of Rayleigh and Love waves on the horizontal components (3cMSPAC; Köhler et al., 2007) and to analyze noise directionality through array beamforming (Ohrnberger et al., 2004). However, the minimum inter-station spacing must be carefully adapted to the frequency range to be resolved. Since our array geometries were designed to detect and locate calving events between 1 and $10 \mathrm{~Hz}$, we cannot use these array methods due to spatial aliasing and lacking wave field correlation at frequencies higher than $10 \mathrm{~Hz}$. A more adequate station setup would potentially allow differentiating between effects of changes in Love wave contribution, noise sources, and propagation medium on HVSR variability. We tried ambient noise interferometry between our array stations as well. However, we encountered lack of waveform correlation due to too-large inter-station distances and locally uncorrelated noise at frequencies higher than $10 \mathrm{~Hz}$. Hence, no seasonal velocity changes related to the active layer could be measured as successfully done by James et al. (2017).

Utilizing a localized and repeating seismic signal for permafrost monitoring might be an alternative to ambient noise HVSRs. The seasonal variations observed in our tremor RVSRs could be either due to changes in the propagation medium or the tremor source itself. In general, the HVSR method is supposed to remove source effects. In our case, for example, the tremor source magnitude variability should affect the vertical and radial components of the Rayleigh wave measured at KBS in the same way. However, we cannot fully exclude the possibility that noise not related to the tremor increases more on the horizontal components during summer than on the vertical component. If the RVSR variability is due to medium changes, the active permafrost layer is a good candidate to explain our observations, though the strongest amplitude increase is expected at much higher frequencies (Fig. 4). Nevertheless, modeling Rayleigh wave ellipticities shows that the tremor frequency band is slightly affected. We obtain a clear increase in ellipticity for the first and second higher modes above $4.5 \mathrm{~Hz}$ for a model assuming very low $S$-wave velocities in the active layer (Table 1, Fig. 7a). This is consistent with Guéguen et al. (2017), who observed a significant HVSR amplitude change within the same frequency band $(2-10 \mathrm{~Hz})$ caused by a $0.75 \mathrm{~m}$ deep frozen layer. However, we cannot exactly reproduce our measured RVSR change due to lacking knowledge about the relative contribution of Rayleigh waves modes and possibly body waves, as well as probably deviations from a 1-D subsurface structure that exist due to dipping layer in the study area (Haldorsen and Heim, 1999). Modeling ellipticities using 2-D or 3-D structures might help to better explain our observations. 
The presence of a repeating, localized tremor signal at higher frequencies around the HVSR peak directly related to the unfrozen layer in summer would allow us to asses the seasonality with higher certainty through directly measuring the peak's frequency change. This potential has to be followed up with more related studies in the future.

Ambient noise and tremor HVSRs complement each other in our case study. The gliding HVSR peak frequency can only be measured from a short record (temporary network), while a long-term record is available for KBS to analyze interannual variability. However, since a permanent station within a shelter structure such as KBS might not be sensitive enough to active layer variability at high frequencies or have a too-low sampling frequency, signals with longer wavelengths are needed. Analyzing the tremor signal allows measuring HVSR variability at lower frequencies that would otherwise (i.e., with ambient noise) not be sensitive enough to resolve active layer thawing. Although the measured quantities are different, ambient noise HVSR peak frequencies and tremor HVSR rms values exhibit consistent variability during the measurement period (see Fig. 8), presumably related to the same cause, i.e., the active permafrost layer.

\section{Summary and recommendations}

We apply the HVSR method to a temporary seismic deployment and the permanent station KBS in northwest Svalbard to investigate its applicability for permafrost active layer monitoring. As expected, ambient noise HVSR variability is strongly affected by changing external site conditions but also reveals a seasonal trend. A gliding peak frequency between 50 and $15 \mathrm{~Hz}$ is observed, which most likely indicates a deepening thaw depth from June until September, as confirmed by modeled HVSRs using the diffuse wave field assumption. Furthermore, we describe a repeating ocean-swelland tide-related seismic tremor in the record of KBS. We are able to extract the frequency-dependent ellipticity from the tremor radial-to-vertical spectral ratios. We find a significant seasonal variation between 4.5 and $5.5 \mathrm{~Hz}$. Although these frequencies are less sensitive to shallow medium changes, we show that Rayleigh wave ellipticities are still affected by the thawed permafrost active layer.

Our results demonstrate that active layer monitoring would benefit from more purpose-built seismic networks and that interpretation of spectral ratio variability must be done carefully to exclude non-structural effects. We confirm previous general recommendations and known issues of the HVSR method (Chatelain et al., 2008), which have become even more important at the high frequencies needed to resolve the active layer HVSR peak. In summary, we suggest the following recommendations, including and emphasizing those given previously and being of special relevance for future passive seismic experiments that have the goal to measure permafrost active layer variability:
1. The seismic sampling rate should be at least $200 \mathrm{~Hz}$ to capture HVSR peaks of shallow, emerging interfaces and to avoid misinterpretation of apparent peaks close to the Nyquist frequency.

2. If logistically feasible, repeated maintenance at temporarily deployed instruments during the melt season is strongly recommended to keep ground coupling stable. Digging instruments deeper into the soil (if deployment is done during thawed conditions) and/or using Posthole sensors, if affordable, is an alternative (Abbott et al., 2016). Cementing the sensor a few decimeters below the surface on a small plate might be another option (Chatelain et al., 2008).

3. A careful evaluation of HVSR variability caused by non-structural effects (e.g., Chatelain et al., 2008) must be performed, for example, using co-located wind speed measurements. As noted in previous studies, time periods with strong wind noise should be excluded from analysis and/or an efficient wind shielding should be used.

4. The deployment of small-aperture seismic arrays with minimum four elements and with minimum inter-station distances not larger than 5 to $10 \mathrm{~m}$ is recommended to allow

a. measuring the frequency- and time-dependent contribution of Rayleigh and Love waves at high frequencies (3c-SPAC method) since a change would affect HVSR amplitudes (Bonnefoy-Claudet et al., 2008);

b. measuring changing noise source directionality and resulting effects on HVSRs (back-azimuth measurements with beamforming/FK analysis);

c. combining HVSR measurements with seismic noise interferometry (James et al., 2017); and

d. comparison and evaluation of HVSRs of close stations affected by more similar local noise and ground conditions.

5. Making use of repeating directional noise sources if applicable has the potential to avoid source variability affecting the HVSRs. If the frequency content of such a source is too low, temporal HVSR increase might still be connected to a peak at higher frequencies. In addition, a purpose-built linear seismic array aligned with propagation direction would allow the application of noise interferometry.

HVSR analysis cannot yet be considered to be a standalone tool to measure permafrost active layer variability without including seismic expert knowledge and taking into account site-dependent factors. However, our study clearly shows the potential of the HVSR method. We are confident 
that more case studies, long-term experiments, and improved instrumental setups will help to establish this approach as a useful supplementary tool in permafrost research.

Data availability. Data of station KBS are freely available through IRIS (ASL/USGS, 1988). The seismic record of the temporary network stations will become freely accessible through the Geophysical Instrument Pool Potsdam (GIPP) after 1 October 2020 (http://gipp.gfz-potsdam.de/webapp/projects/view/536, GFZ, 2019). Measured sea level data from Ny-Ålesund were obtained from https://www.kartverket.no/en/sehavniva/Lokasjonsside/ ?cityid=9000015\&city=Ny-C385lesund (Kartverket, 2019). Meteorological data are available from re3data.org (2018) and soil temperatures at station Bayelva from Boike et al. (2017). Copernicus Sentinel data from 2016 were used in Fig. 1. 
Appendix A: Supporting figures for HVSRs from ambient seismic noise

Figure A1 shows examples of deployed seismic sensors. Figures $\mathrm{A} 2$ and $\mathrm{A} 3$ show the HVSRs for the stations not shown in the main text.

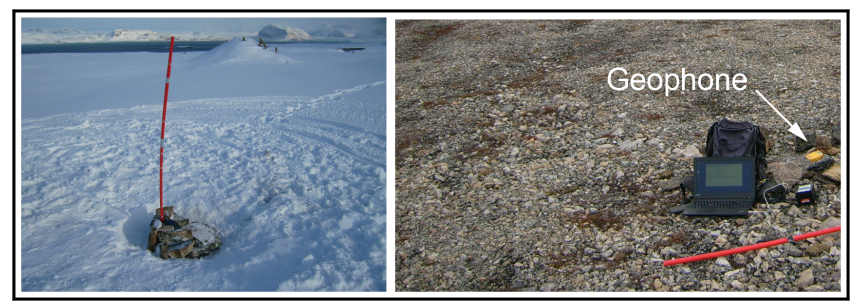

Figure A1. Photos of a station of the KBSA array after deployment in April and a station of the BRA array during data retrieval in August (geophone uncovered).

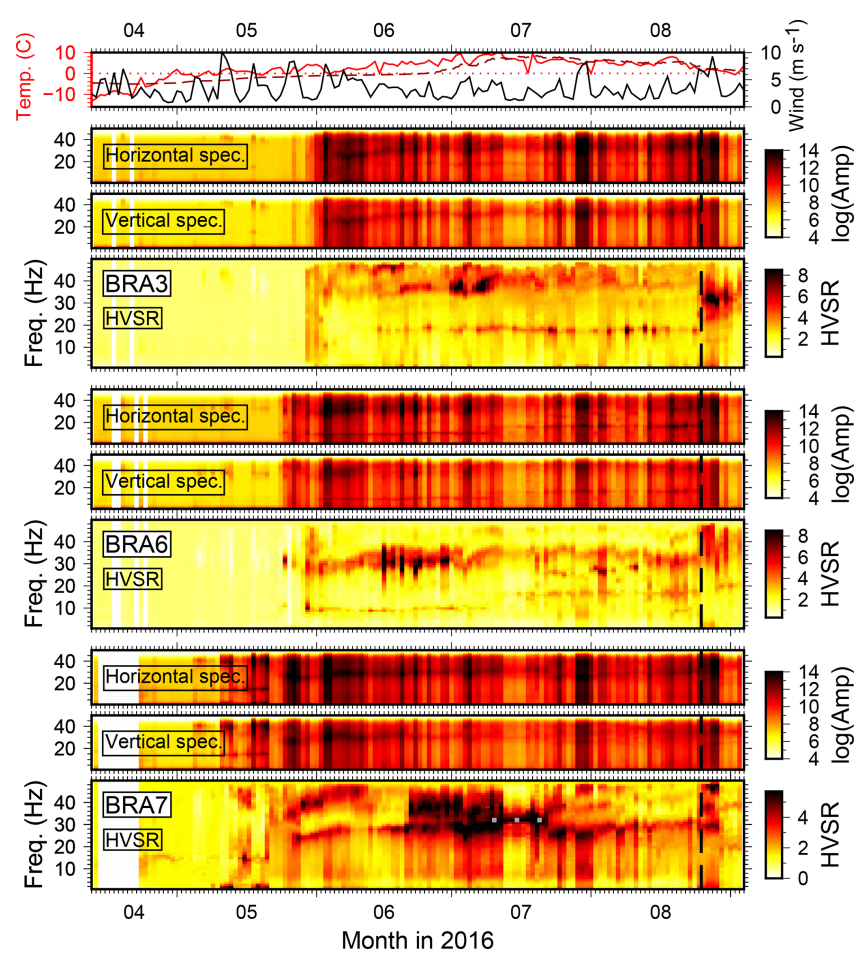

Figure A2. Same as Fig. 2 for three more stations.

\section{Appendix B: Automatic detection and temporal distribution of the repeating tremor}

Repeating tremors in the KBS record are detected using a STA/LTA trigger applied to a time series of vertical component spectral amplitudes. We compute the logarithm of spectral power between 3.4 and $5.7 \mathrm{~Hz}$ in non-overlapping $150 \mathrm{~s}$ long time windows. A STA length of $25 \mathrm{~min}$, a LTA length

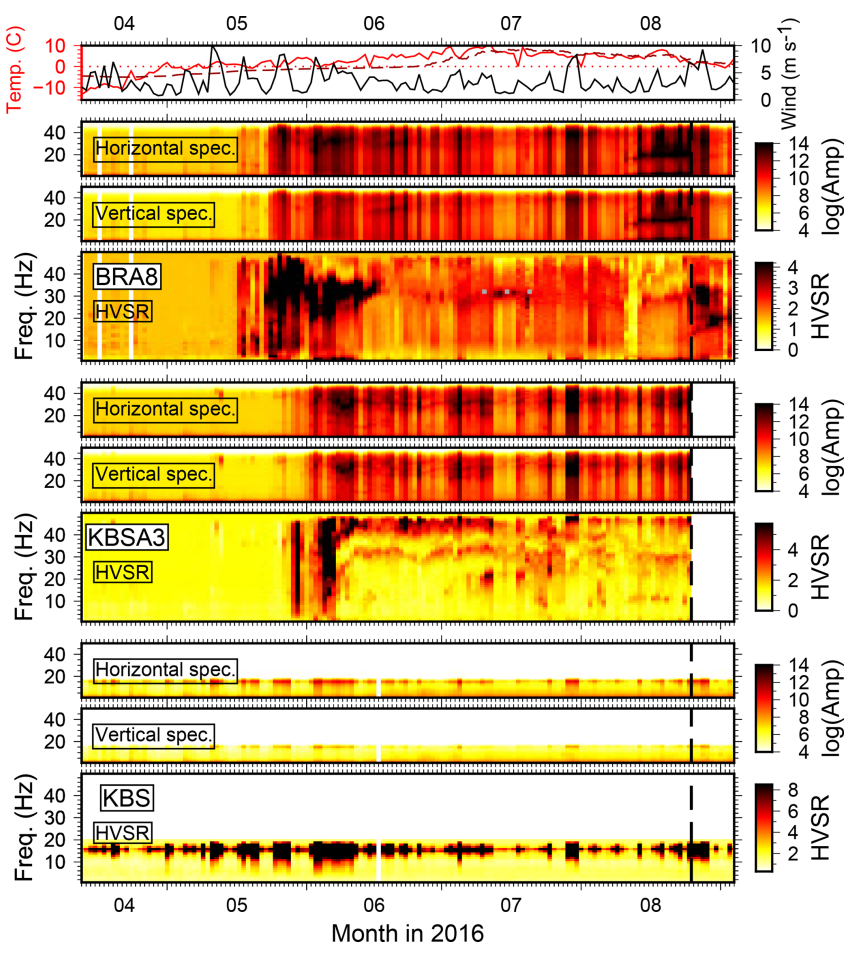

Figure A3. Same as Fig. 2 for three more stations.

of $25 \mathrm{~h}$, and a STA/LTA threshold of 1.15 are used. If the threshold is exceeded for a sample (time window), the occurrence of a tremor is declared. Samples are assigned to the same tremor if gaps between exceeded thresholds are shorter than $1 \mathrm{~h}$. If the gap is longer, the onset of a new tremor is declared. Detections with duration less than $25 \mathrm{~min}$ are sorted out. All detection parameters are found by evaluating if clear, visually identified tremors are correctly detected, while minimizing the number of false detections. Visual post-processing is done to reject a few false positives so that only real tremors are used for further processing. The list of all detected tremors is provided in the Supplement S02. Tremors were detected around semi-diurnal tidal maxima in Ny-Ålesund (Fig. 5), except during neap tides and at low wind speed. Sometimes two tremors are declared if the amplitudes exhibit a two-sided distribution, i.e., peaks at the start and the end of a tremor (see, for example, 26 January 2008 and 28 January 2016 in Fig. 5a). The amplitude spectrum of the time series of log-spectral powers used for the detector shows prominent semi-diurnal tidal peaks (Fig. B2, Darwin symbols of tides: $\mathrm{M}_{2}, \mathrm{~S}_{2}, \mathrm{~N}_{2}$ ). Furthermore, diurnal $\left(\mathrm{K}_{1}, \mathrm{O}_{1}\right)$, terci-diurnal $\left(\mathrm{M}_{3}\right)$, and quarti-diurnal $\left(\mathrm{M}_{4}\right)$ peaks are clearly revealed. The neap-spring tide cycle (14.75 days, $\mathrm{M}_{\mathrm{sf}}$ ) appears as a weak peak in the spectrum. In some years $(2003,2004,2009-2011)$, the number of tremor detections drops in the beginning of the year, which could be an effect of sea ice preventing ocean wave activity. Note that in recent years (from about 2013), no land-fastened sea 


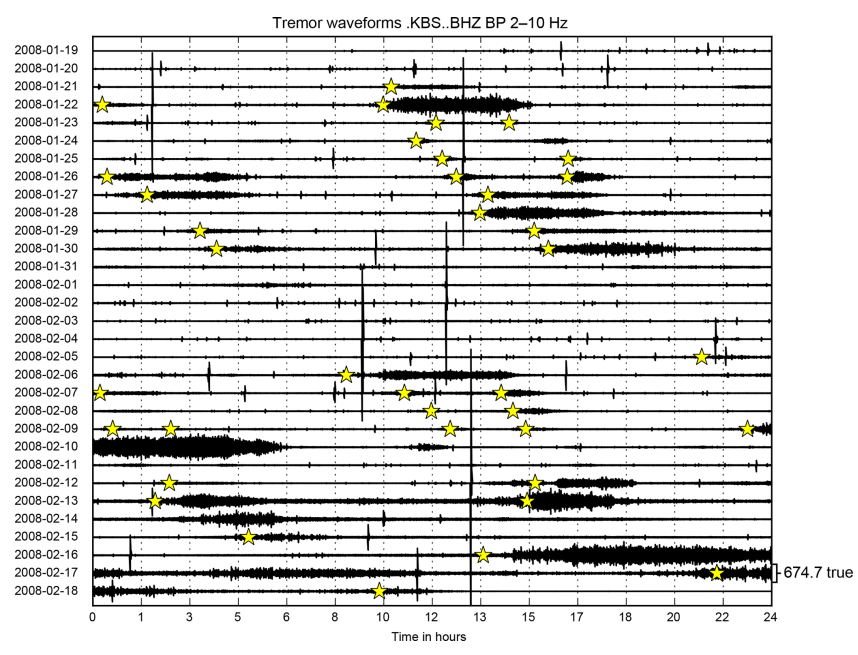

Figure B1. Example of repeating seismic tremor waveforms recorded at KBS. Detected tremor onsets are indicated by yellow stars. Waveform data of the tremor on 22 January 2008 are provided in the Supplement S01.

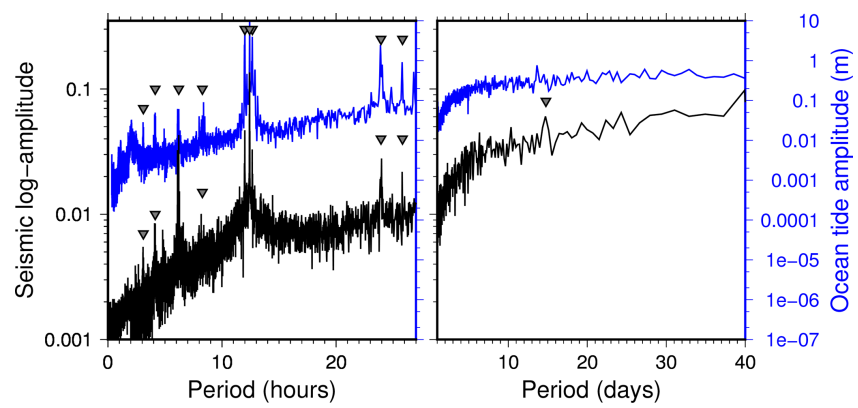

Figure B2. Amplitude spectrum of time series of log-spectral powers of KBS vertical component seismic data (see Fig. 5a) and of measured sea level in Ny-Ålesund between 2005 and 2016. Gray triangles indicate theoretical ocean tide periods at (from left to right, Darwin symbol of tide in brackets) $3.105 \mathrm{~h}\left(\mathrm{M}_{8}\right), 4.14 \mathrm{~h}\left(\mathrm{M}_{6}\right)$, $6.21 \mathrm{~h}\left(\mathrm{M}_{4}\right), 8.28 \mathrm{~h}\left(\mathrm{M}_{3}\right), 12.0 \mathrm{~h}\left(\mathrm{~S}_{2}\right), 12.42 \mathrm{~h}\left(\mathrm{M}_{2}\right), 12.658 \mathrm{~h}\left(\mathrm{~N}_{2}\right)$, $23.93 \mathrm{~h}\left(\mathrm{~K}_{1}\right), 25.82 \mathrm{~h}\left(\mathrm{O}_{1}\right)$, and 14.75 days $\left(\mathrm{M}_{\mathrm{sf}}\right)$.

ice has been observed at the coast of Ny-Ålesund (personal communication, Christopher Nuth, 2018).

\section{Appendix C: Supporting figures for tremor spectrum and polarization}

Figure $\mathrm{C} 1$ shows results of $\mathrm{FK}$ analysis for a tremor record. Measured back-azimuth at KBSA array and $P$-wave polarization angle for regional earthquakes are shown in Fig. C2.

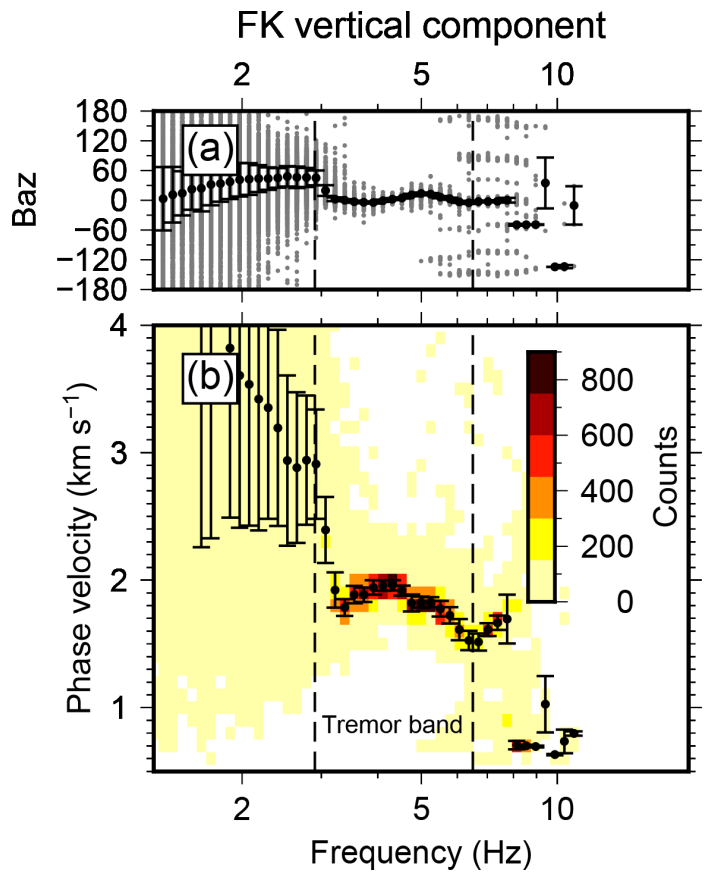

Figure C1. Example of FK analysis of vertical components of KBSA array for a tremor occurring between 12 May 2016 03:02:00 UTC and 12 May 2016 06:17:00 UTC. (a) All backazimuth (baz) measurements at maximum beam power and with coherency (normalized beam power) $>0.7$ for $600 \mathrm{~s}$ long time windows during tremor occurrence (gray symbols) and median with median deviation (black). (b) Color-coded histogram (counts) of phase velocity measurements for same time windows as in panel (a) and median with median deviation.

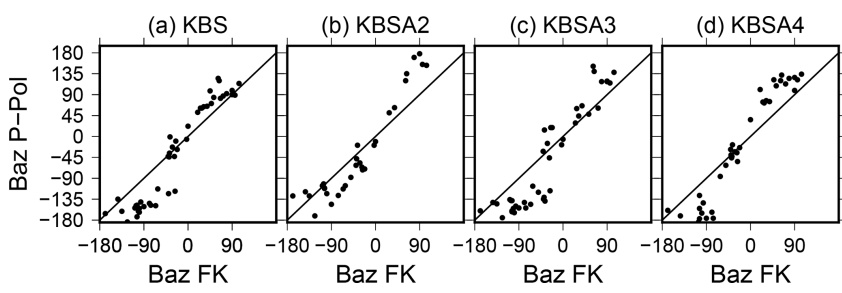

Figure C2. Back-azimuth measured with FK analysis at KBSA array vs. station's $P$-wave polarization angle measured from regional earthquakes. 
Supplement. The supplement related to this article is available online at: https://doi.org/10.5194/esurf-7-1-2019-supplement.

Author contributions. AK and CW initiated the study. AK processed and analyzed the seismic data and prepared the manuscript. $\mathrm{CW}$ was responsible for field instrumentation and assisted in the field experiment and manuscript editing.

Competing interests. The authors declare that they have no conflict of interest.

Special issue statement. This article is part of the special issue "From process to signal - advancing environmental seismology". It is a result of the EGU Galileo conference, Ohlstadt, Germany, 6-9 June 2017.

Acknowledgements. This study was financed by the Norwegian Research Council funded CalvingSEIS (244196/E10) and SEISMOGLAC (213359/F20) projects. Seismic instrumentation for temporary network was provided by the Geophysical Instrument Pool of GFZ Potsdam, Germany. Special thanks go to Christopher Nuth (PI of CalvingSEIS) for organizing logistics in Ny-Ålesund and for helping, together with Cesar DeschampsBerger, during instrument deployment. We used ObsPy (Beyreuther et al., 2010) for seismic data analysis. Figures were produced using GMT (Wessel and Smith, 1998). Rayleigh wave ellipticities were computed using Geopsy (http://www.Geopsy.org, last access: 7 January 2019). We thank Antonio Garcia Jerez for providing us with the HV-Inv software to model HVSRs using the diffuse wave field theory. We thank Lukas Preiswerk, Philippe Guéguen, and one anonymous reviewer for reviewing the manuscript.

Edited by: Fabian Walter

Reviewed by: Lukas Preiswerk and one anonymous referee

\section{References}

Abbott, R., Knox, H. A., James, S., Lee, R., and Cole, C.: Permafrost Active Layer Seismic Interferometry Experiment (PALSIE), Tech. rep., Sandia National Laboratories (SNL-NM), Albuquerque, NM (United States), available at: https://prod. sandia.gov/techlib/access-control.cgi/2016/160167.pdf (last access: 7 January 2019), 2016.

Adams, P. N., Anderson, R. S., and Revenaugh, J.: Microseismic measurement of wave-energy delivery to a rocky coast, Geology, 30, 895-898, https://doi.org/10.1130/00917613(2002)030<0895:MMOWED>2.0.CO;2, 2002.

Albuquerque Seismological Laboratory (ASL)/USGS: Global Seismograph Network (GSN - IRIS/USGS), https://doi.org/10.7914/sn/iu, 1988.

Bartholomaus, T. C., Amundson, J. M., Walter, J. I., O’Neel, S., West, M. E., and Larsen, C. F.: Subglacial discharge at tidewater glaciers revealed by seismic tremor, Geophys. Res. Lett., 42, 6391-6398, https://doi.org/10.1002/2015GL064590, 2015.
Beyreuther, M., Barsch, R., Krischer, L., Megies, T., Behr, Y., and Wassermann, J.: ObsPy: A Python toolbox for seismology, Seismol. Res. Lett., 81, 530-533, https://doi.org/10.1785/gssrl.81.3.530, 2010.

Boike, J., Juszak, I., Lange, S., Chadburn, S., Burke, E., Overduin, P. P., Roth, K., Ippisch, O., Bornemann, N., Stern, L., Gouttevin, I., Hauber, E., and Westermann, S.: Soil data at station Bayelva (1998-2017, level 2, version 1), https://doi.org/10.1594/PANGAEA.882061, 2017.

Boike, J., Juszak, I., Lange, S., Chadburn, S., Burke, E., Overduin, P. P., Roth, K., Ippisch, O., Bornemann, N., Stern, L., Gouttevin, I., Hauber, E., and Westermann, S.: A 20-year record (19982017) of permafrost, active layer and meteorological conditions at a high Arctic permafrost research site (Bayelva, Spitsbergen), Earth Syst. Sci. Data, 10, 355-390, https://doi.org/10.5194/essd10-355-2018, 2018.

Bonnefoy-Claudet, S., Cotton, F., and Bard, P.-Y.: The nature of noise wavefield and its applications for site effects studies: A literature review, Earth-Sci. Rev., 79, 205-227, https://doi.org/10.1016/j.earscirev.2006.07.004, 2006.

Bonnefoy-Claudet, S., Köhler, A., Cornou, C., Wathelet, M., and Bard, P.-Y.: Effects of Love waves on microtremor H/V ratio, B. Seismol. Soc. Am., 98, 288-300, https://doi.org/10.1785/0120070063, 2008.

Burtin, A., Cattin, R., Bollinger, L., Vergne, J., Steer, P., Robert, A., Findling, N., and Tiberi, C.: Towards the hydrologic and bed load monitoring from high-frequency seismic noise in a braided river: The "torrent de St Pierre", French Alps, J. Hydrol., 408, 43-53, https://doi.org/10.1016/j.jhydrol.2011.07.014, 2011.

Chatelain, J.-L., Guillier, B., Cara, F., Duval, A.-M., Atakan, K., and Bard, P.-Y.: Evaluation of the influence of experimental conditions on $\mathrm{H} / \mathrm{V}$ results from ambient noise recordings, B. Earthq. Eng., 6, 33-74, https://doi.org/10.1007/s10518-0079040-7, 2008.

Cox, B. R., Wood, C. M., and Hazirbaba, K.: Frozen and unfrozen shear wave velocity seismic site classification of Fairbanks, Alaska, J. Cold Reg. Eng., 26, 118-145, https://doi.org/10.1061/(ASCE)CR.1943-5495.0000041, 2012.

Dickson, M. E. and Pentney, R.: Micro-seismic measurements of cliff motion under wave impact and implications for the development of near-horizontal shore platforms, Geomorphology, 151, 27-38, https://doi.org/10.1016/j.geomorph.2012.01.006, 2012.

Earlie, C. S., Young, A. P., Masselink, G., and Russell, P. E.: Coastal cliff ground motions and response to extreme storm waves, Geophys. Res. Lett., 42, 847-854, https://doi.org/10.1002/2014GL062534, 2015.

García-Jerez, A., Piña-Flores, J., Sánchez-Sesma, F. J., Luzón, F., and Perton, M.: A computer code for forward calculation and inversion of the $\mathrm{H} / \mathrm{V}$ spectral ratio under the diffuse field assumption, Comput. Geosci., 97, 67-78, https://doi.org/10.1016/j.cageo.2016.06.016, 2016.

GFZ: GIPP Experiment Database, available at: http://gipp. gfz-potsdam.de/webapp/projects/view/536, last access: 7 January 2019.

Guéguen, P., Langlais, M., Garambois, S., Voisin, C., and DousteBacqué, I.: How sensitive are site effects and building response to extreme cold temperature? The case of the Grenoble's (France) City Hall building, B. Earthq. Eng., 15, 889-906, https://doi.org/10.1007/s10518-016-9995-3, 2017. 
Haldorsen, S. and Heim, M.: An Arctic groundwater system and its dependence upon climatic change: an example from Svalbard, Permafrost Periglac., 10, 137-149, 1999.

Haldorsen, S., Heim, M., and Lauritzen, S.-E.: Subpermafrost groundwater, western Svalbard: Paper presented at the 10th Northern Res. Basin Symposium (Svalbard, Norway - 28 Aug./3 Sept. 1994), Hydrol. Res., 27, 57-68, https://doi.org/10.2166/nh.1996.0019, 1996.

James, S., Knox, H., Abbott, R., and Screaton, E.: Improved moving window cross-spectral analysis for resolving large temporal seismic velocity changes in permafrost, Geophys. Res. Lett., 44, 4018-4026, https://doi.org/10.1002/2016GL072468, 2017.

Jones, E. V., Rosser, N., Brain, M., and Petley, D.: Quantifying the environmental controls on erosion of a hard rock cliff, Mar. Geol., 363, 230-242, https://doi.org/10.1016/j.margeo.2014.12.008, 2015.

King, M., Zimmerman, R., and Corwin, R.: Seismic and electrical properties of unconsolidated permafrost, Geophys. Prospect., 36, 349-364, https://doi.org/10.1111/j.1365-2478.1988.tb02168.x, 1988.

Köhler, A., Ohrnberger, M., and Scherbaum, F.: The relative fraction of Rayleigh and Love waves in ambient vibration wavefields at different European sites, in: Proceedings of the third International Symposium on the Effects of Surface Geology on Seismic Motion, Grenoble, France, Paper Number/Abstract ID: 83, 2006.

Köhler, A., Ohrnberger, M., Scherbaum, F., Wathelet, M., and Cornou, C.: Assessing the reliability of the modified three-component spatial autocorrelation technique, Geophys. J. Int., 168, 779-796, https://doi.org/10.1111/j.1365246X.2006.03253.x, 2007.

Kartverket: Water level and tidal information Ny-Ålesund, available at: https://www.kartverket.no/en/sehavniva/Lokasjonsside/ ?cityid $=9000015 \&$ city $=\mathrm{Ny}-\% \mathrm{C} 3 \% 85$ lesund , last access: 7 January 2019.

Köhler, A., Nuth, C., Schweitzer, J., Weidle, C., and Gibbons, S. J.: Regional passive seismic monitoring reveals dynamic glacier activity on Spitsbergen, Svalbard, Polar Res., 34, 26178, https://doi.org/10.3402/polar.v34.26178, 2015.

Köhler, A., Nuth, C., Kohler, J., Berthier, E., Weidle, C., and Schweitzer, J.: A 15 year record of frontal glacier ablation rates estimated from seismic data, Geophys. Res. Lett., 43, 1215512164, https://doi.org/10.1002/2016GL070589, 2016.

Kula, D., Olszewska, D., Dobiński, W., and Glazer, M.: Horizontal-to-vertical spectral ratio variability in the presence of permafrost, Geophys. J. Int., 214, 219-231, https://doi.org/10.1093/gji/ggy118, 2018.

Kvaerna, T. and Ringdal, F.: Stability of various fk estimation techniques, in: NORSAR Semiannual technical summary, 29-40, NORSAR Scientific Report 1-86/87, 1986.

Lachet, C. and Bard, P.-Y.: Numerical and theoretical investigations on the possibilities and limitations of Nakamura's technique, J. Phys. Earth, 42, 377-397, https://doi.org/10.4294/jpe1952.42.377, 1994.

Larose, E., Carrière, S., Voisin, C., Bottelin, P., Baillet, L., Guéguen, P., Walter, F., Jongmans, D., Guillier, B., Garambois, S., Florent, G., and Chris, M.: Environmental seismology: What can we learn on earth surface processes with ambient noise?, J. Appl. Geophys., 116, 62-74, https://doi.org/10.1016/j.jappgeo.2015.02.001, 2015.
LeBlanc, A.-M., Fortier, R., Allard, M., Cosma, C., and Buteau, S.: Seismic cone penetration test and seismic tomography in permafrost, Can. Geotech. J., 41, 796-813, https://doi.org/10.1139/t04-026, 2004.

Lévêque, J.-J., Maggi, A., and Souriau, A.: Seismological constraints on ice properties at Dome C, Antarctica, from horizontal to vertical spectral ratios, Antarct. Sci., 22, 572-579, https://doi.org/10.1017/S0954102010000325, 2010.

Lunedei, E. and Malischewsky, P.: A review and some new issues on the theory of the H/V technique for ambient vibrations, in: Perspectives on European Earthquake Engineering and Seismology, 371-394, Springer, https://doi.org/10.1007/978-3-31916964-4_15, 2015.

Nakamura, Y.: A method for dynamic characteristics estimation of subsurface using microtremor on the ground surface, QR Railway Tech. Res. Inst., 30, 25-33, 1989.

Neuffer, T. and Kremers, S.: How wind turbines affect the performance of seismic monitoring stations and networks, Geophys. J. Int., 211, 1319-1327, https://doi.org/10.1093/gji/ggx370, 2017.

Norman, E. C., Rosser, N. J., Brain, M. J., Petley, D. N., and Lim, M.: Coastal cliff-top ground motions as proxies for environmental processes, J. Geophys. Res.-Oceans, 118, 6807-6823, https://doi.org/10.1002/2013JC008963, 2013.

Ohrnberger, M., Schissele, E., Cornou, C., Bonnefoy-Claudet, S., Wathelet, M., Savvaidis, A., Scherbaum, F., and Jongmans, D.: Frequency wavenumber and spatial autocorrelation methods for dispersion curve determination from ambient vibration recordings, in: Proceedings of the 13th World Conference on Earthquake Engineering, 946, available at: http://www.iitk.ac.in/nicee/ wcee/article/13_946.pdf (last access: 7 January 2019), 2004.

Overduin, P. P., Haberland, C., Ryberg, T., Kneier, F., Jacobi, T., Grigoriev, M., and Ohrnberger, M.: Submarine permafrost depth from ambient seismic noise, Geophys. Res. Lett., 42, 7581-7588, https://doi.org/10.1002/2015GL065409, 2015.

Parolai, S., Picozzi, M., Richwalski, S., and Milkereit, C.: Joint inversion of phase velocity dispersion and $\mathrm{H} / \mathrm{V}$ ratio curves from seismic noise recordings using a genetic algorithm, considering higher modes, Geophys. Res. Lett., 32, L01303, https://doi.org/10.1029/2004GL021115, 2005.

Picotti, S., Francese, R., Giorgi, M., Pettenati, F., and Carcione, J. M.: Estimation of glacier thicknesses and basal properties using the horizontal-to-vertical component spectral ratio (HVSR) technique from passive seismic data, J. Glaciol., 63, 229-248, https://doi.org/10.1017/jog.2016.135, 2017.

re3data.org: eKlima, https://doi.org/10.17616/R3Q63H, 2018.

Sabra, K., Gerstoft, P., Roux, P., Kuperman, W., and Fehler, M.: Extracting time-domain Green's function estimates from ambient seismic noise, Geophys. Res. Lett., 32, L03310, https://doi.org/10.1029/2004GL021862, 2005.

Saccorotti, G., Piccinini, D., Cauchie, L., and Fiori, I.: Seismic noise by wind farms: a case study from the Virgo Gravitational Wave Observatory, Italy, B. Seismol. Soc. Am., 101, 568-578, https://doi.org/10.1785/0120100203, 2011.

Sánchez-Sesma, F. J.: Modeling and inversion of the microtremor $\mathrm{H} / \mathrm{V}$ spectral ratio: physical basis behind the diffuse field approach, Earth Planets Space, 69, 92, https://doi.org/10.1186/s40623-017-0667-6, 2017.

Sens-Schönfelder, C. and Wegler, U.: Passive image interferometry and seasonal variations of seismic velocities at Mer- 
api Volcano, Indonesia, Geophys. Res. Lett., 33, L21302, https://doi.org/10.1029/2006GL027797, 2006.

Sens-Schönfelder, C. and Wegler, U.: Passive image interferometry for monitoring crustal changes with ambient seismic noise, C. R. Geosci., 343, 639-651, https://doi.org/10.1016/j.crte.2011.02.005, 2011.

Shapiro, N. and Campillo, M.: Emergence of broadband Rayleigh waves from correlations of the ambient seismic noise, Geophys. Res. Lett., 31, 1615-1619, https://doi.org/10.1029/2004GL019491, 2004.

Snieder, R.: The theory of coda wave interferometry, Pure Appl. Geophys., 163, 455-473, https://doi.org/10.1007/s00024-0050026-6, 2006

van der Ploeg, M. J., Haldorsen, S., Leijnse, A., and Heim, M.: Subpermafrost groundwater systems: Dealing with virtual reality while having virtually no data, J. Hydrol., 475, 42-52, https://doi.org/10.1016/j.jhydrol.2012.08.046, 2012.

Wessel, P. and Smith, W. H. F.: New, improved version of GMT released, EOS T. Am. Geophys. Un., 79, 579-579, https://doi.org/10.1029/98EO00426, 1998.
Westermann, S., Wollschläger, U., and Boike, J.: Monitoring of active layer dynamics at a permafrost site on Svalbard using multichannel ground-penetrating radar, The Cryosphere, 4, 475-487, https://doi.org/10.5194/tc-4-475-2010, 2010.

Xu, G., Yang, Z., Dutta, U., Tang, L., and Marx, E.: Seasonally frozen soil effects on the seismic site response, J. Cold Reg. Eng., 25, 53-70, https://doi.org/10.1061/(ASCE)CR.19435495.0000022, 2010.

Yan, P., Li, Z., Li, F., Yang, Y., Hao, W., and Bao, F.: Antarctic ice sheet thickness estimation using the horizontal-to-vertical spectral ratio method with single-station seismic ambient noise, The Cryosphere, 12, 795-810, https://doi.org/10.5194/tc-12795-2018, 2018.

Young, A. P., Guza, R. T., O'Reilly, W. C., Burvingt, O., and Flick, R. E.: Observations of coastal cliff base waves, sand levels, and cliff top shaking, Earth Surf. Proc. Land., 41, 1564-1573, https://doi.org/10.1002/esp.3928, 2016. 